\title{
Dosimetric Evaluation of Intensity Modulated Radiotherapy and Three-Dimensional Conformal Radiotherapy Treatment Plans for Prostate Cancer
}

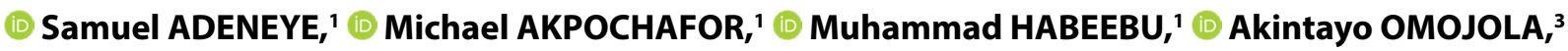 \\ (1) Adedayo JOSEPH, ${ }^{2}$ (D) John AWHARIADO, ${ }^{\circledR}$ Abayomi DUROSINNMI-ETTI, ${ }^{2}$ (D) Moses AWEDA'
}

'Department of Radiation Biology, Radiotherapy and Radiodiagnosis, College of Medicine, University of Lagos, Lagos-Nigeria ${ }^{2}$ Department of Radiotherapy, Nigerian Sovereign Investment Authority-Lagos University Teaching Hospital, Lagos-Nigeria ${ }^{3}$ Department of Radiology, Federal Medical Centre Asaba, Medical Physics Unit, Asaba-Nigeria

\begin{abstract}
OBJECTIVE
We evaluated the homogeneity index and conformity index using intensity modulated radiotherapy (IMRT) and three-dimensional conformal radiotherapy (3D-CRT) treatment plans in prostate cancer.

\section{METHODS}

Twenty treatment plans for ten patients were created using 3D-CRT of four-fields with gantry angles of $0^{\circ}, 90^{\circ}, 180^{\circ}$, and $270^{\circ}$; and IMRT of five-fields with gantry angles of $0^{\circ}, 72^{\circ}, 144^{\circ}, 216^{\circ}$, and $288^{\circ}$ on an Eclipse Treatment Planning System (version 15.6). The volume of reference isodose, target volume, maximum isodose in the target, reference isodose, dose at $95 \%$ of planning target volume (PTV), dose at $2 \%, 5 \%$, and $98 \%$ of PTV, and prescribed dose were collected from the dose volume histogram of each plan. The conformity index and homogeneity index (HI) were then calculated. The doses of the organs at risk were also collected and evaluated.
\end{abstract}

\section{RESULTS}

The HI of the twenty patients who underwent the treatment plan with 3D-CRT was $1.088 \pm 0.03$, which shows good homogeneity, but less homogeneity when compared with plans done with IMRT (1.072 \pm 0.02$)$.

\section{CONCLUSION}

The use of IMRT treatment plan for prostate cancer proved to be superior over 3D-CRT in terms of conformity and homogeneity, as well as sparring dose to organ at risk.

Keywords: Conformity index; homogeneity index; intensity modulated radiotherapy; prostate cancer; 3D-conformal radiotherapy.

Copyright $\odot$ 2021, Turkish Society for Radiation Oncology

\section{Introduction}

Till date, cancer is among the most feared diseases with high mortality rate. Consistence with this, an estimated number of new cancers were diagnosed in 2019 in the United State as 1.762 .450 , with a total of 606.880 deaths recorded.[1] In Nigeria, according to the International Agency on Research on Cancer, as of 2018, the total number of new cases was 115.950 , with 70.327 deaths recorded.[2] Prostate cancer is the second leading cause of cancer in men. In 2019, a total of 174.650 men were diagnosed with prostate cancer in the United 
States (cancer.net) and, in Nigeria, an estimated hospital prevalence of between 127 and 185.5 per 100.000 males admitted in hospitals were diagnosed of prostate cancer.[3] Prostate cancer can be treated by surgery, radiation therapy, chemotherapy, cryotherapy, hormone therapy and immunotherapy, and newer technological development.[4] Radiation therapy has a dynamic role in the treatment of prostate cancer. It involves the use of various treatment plans (TPs) such as $2 \mathrm{D}$ - technique, 3D-Conformal Radiation Therapy (3D-CRT), and intensity modulated radiotherapy (IMRT). 2D- technique involves manual calculations and does not spare organs at risk (OAR). 3D-Conformal Radiation Therapy (3DCRT) is a conformal TP that conforms the radiation doses to the target and, in history, was the best TP for prostate cancer, but results to little sparing of OAR. With intensity modulated radiotherapy (IMRT), the reduction of radiation effect on normal tissues has improved. Research has shown that IMRT has more advantages compared to 3D-CRT in the treatment of prostate cancer. In this study, we investigated the use of homogeneity index (HI) and conformity index (CI) in the evaluation of 3DCRT and IMRT plans for optimal treatment delivery.

\section{Materials and Methods}

\section{Patients Selection}

Ten patients with malignant neoplasm of prostate that received radiotherapy with IMRT on a clinical linear accelerator (LINAC), Vitalbeam model (Varian Medical System, Palo Alto, CA, USA) in our department from June 2019 to January 2020 were analyzed, retrospectively.

\section{Simulation and Contouring}

Each patient was asked to stay on a supine position on a whole-body board (Radon Medical Equipment, Yenimahalle/ANKARA) without immobilization and was simulated with a 16-slice computed tomography (CT) simulator (Optima 580; GE Healthcare, Waukesha, WI, USA). The plans were sequentially done in three phases. The clinical treatment volume (CTV) for one of the cases was contoured in two phases and nine cases were contoured in three phases. Each planning target volume (PTV) was contoured with $0.5 \mathrm{~cm}$ margin from each CTV. Phase 1 ( $\mathrm{PH} \mathrm{1)}$ contains the prostate, seminal vesicle, and lymph node. Phase 2 ( $\mathrm{PH} 2$ ) contains the prostate and seminal vesicle only, while phase 3 (PH 3) contains the prostate only. However, the case with two phases had phase 1 (the prostate+seminal vesicle+lymph node) and phase 2 (the prostate only)
(Table 1). The OARs, which are rectum, bladder, and femoral heads (left and right), were also contoured according to the Radiation Therapy Oncology Group (RTOG) atlas for contouring of normal tissue [5] using the Eclipse TP system version 15.6.

\section{TPs}

Two plans were generated for each patient using the Eclipse TP system version 15.6, with energy of 6 MV photons. The prescribed dose was as follows: 76 Gy for three cases; 79 Gy for six cases; and 69 Gy for the patient planned in two phases as shown in Table 1. The different prescription was due to the different non-use of uniform prescription model in our center. The oncologist's prescription type depended on the cancer stage. Each 3D-CRT plan was produced using four beams (box technique) at the gantry angles of $0^{\circ}$, $90^{\circ}, 180^{\circ}$, and $270^{\circ}$. Multi-leaf collimators (MLC 120 model) were used at $0.5 \mathrm{~cm}$ away from PTV to reduce dose to OAR and for more conformity of the 3D-CRT plans. The IMRT plans were done using five beams at the gantry angles of $0^{\circ}, 72^{\circ}, 144^{\circ}, 216^{\circ}$, and $288^{\circ}$. The intensity optimization for each of the beam portals for all IMRT plans was achieved by setting dose constraints and priorities for PTV and OAR until the constraints were met, following the International Commission on Radiation Units and Measurement (ICRU) protocol for dose prescription, with a minimum coverage dose of $95 \%$ and maximum accepted dose of $107 \%$.[6] The doses were calculated using Anisotropic Analyses Algorithm in the Eclipse TP system, with the treatment table or couch not included in the calculation volume.

When creating the IMRT plan for a LINAC equipped with an MLC, there were two delivery options: stepand-shoot and sliding window. For this study, the sliding window was adopted for all the IMRT plans.

The Quantitative Analysis of Normal Tissue Effects in the Clinic (QUANTEC) analysis and Radiation Therapy Oncology Group (RTOG) Report 62 (a review of Report 50) guideline were adopted for the dose constraint reaching the OAR. The guideline stipulates that

\begin{tabular}{|c|c|c|c|}
\hline Table 1 & $\begin{array}{l}\text { Showing the } \\
\text { tients for bot }\end{array}$ & $\begin{array}{l}\text { ibed doses } \\
\text { RT and IMF }\end{array}$ & e ten pa- \\
\hline Phases & $69 \mathrm{~Gy}$ & $76 \mathrm{~Gy}$ & $79 \mathrm{~Gy}$ \\
\hline PH 1 & $45 \mathrm{~Gy} / 25 \mathrm{fr}$ & $46 \mathrm{~Gy} / 23 \mathrm{fr}$ & $45 \mathrm{~Gy} / 25 \mathrm{fr}$ \\
\hline $\mathrm{PH} 2$ & $24 \mathrm{~Gy} / 12 \mathrm{fr}$ & $10 \mathrm{~Gy} / 5 \mathrm{fr}$ & $9 \mathrm{~Gy} / 5 \mathrm{fr}$ \\
\hline $\mathrm{PH} 3$ & & $20 \mathrm{~Gy} / 10 \mathrm{fr}$ & $25 \mathrm{~Gy} / 14 \mathrm{fr}$ \\
\hline
\end{tabular}

3D-CRT: Three-dimensional conformal radiotherapy; IMRT: Intensity modulated radiotherapy. 
not more than $35 \%$ of the rectum should receive $60 \mathrm{~Gy}$ (V60 Gy <35\%) and not more than $20 \%$ of the rectum should receive 70 Gy (V70 Gy <20\%). Also, for the bladder, not more than $15 \%$ of the bladder should receive 80 Gy (V80 Gy $<15 \%$ ), not more than $25 \%$ should receive 75 Gy (V75 Gy <25\%), not more than 35\% should receive 70 Gy (V70 Gy $<35 \%$ ), and not more than $50 \%$ should receive $60 \mathrm{~Gy}$ (V60 Gy $<50 \%)$. For the femoral heads, not more than $5 \%$ of the femoral heads should receive 50 Gy (V50 Gy <5\%).[5,7-9]

\section{Dose Volume Analysis}

The plan sums for the different plans were generated and data were collected from their dose volume histogram (DVH). From the DVH, the value of dose in Gy reaching the following volume of PTV was recorded: V2\%, V5\%, V50\%, V95\%, and V98\%. Also, the maximum isodose in the target (Imax) and the reference isodose reaching V95\% of PTV were also recorded.

\section{$\mathrm{CI}$ and $\mathrm{HI}$}

$\mathrm{CI}$ and $\mathrm{HI}$ were calculated and recorded for each TP using the following equations: $[10,11]$

$$
\mathbf{C I}=\frac{V_{R I}}{T V}
$$

Where $V_{R I}$ is volume of the target receiving $95 \%$ of the prescribed dose and $T V$ is the total volume of the target.

$\mathrm{HI}\left(\mathrm{H}_{1}\right)=\frac{I_{\max }}{R I}$

Where $\mathrm{I}_{\max }$ is maximum dose in the target RI is reference isodose and

$\mathbf{H}_{2}=\frac{\boldsymbol{D}_{\geq 5 \%} \%}{\boldsymbol{D}_{\geq 95 \%}}$
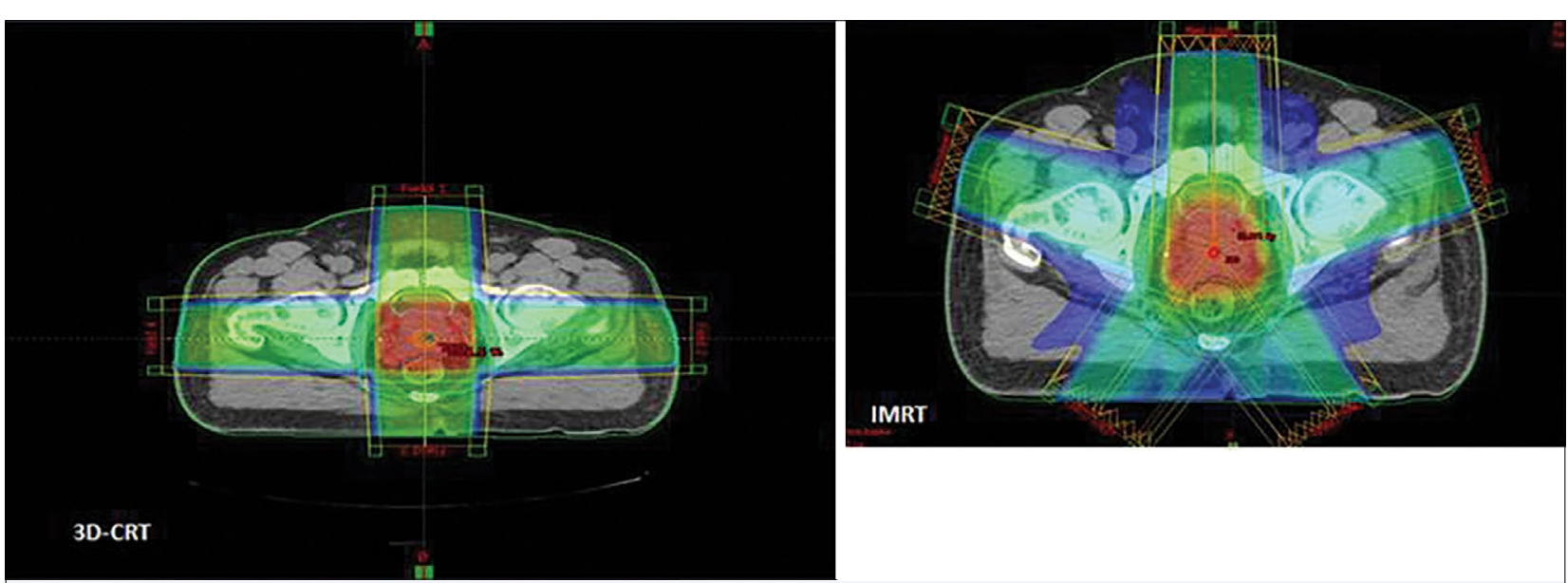

Fig. 1. Showing the dose distribution of a 3D-CRT and IMRT plans for a selected patient.
Where:

$\mathrm{D}_{\geq 95 \%}$ is dose at $95 \%$ of planning target volume $\mathrm{D}_{25 \%}$ is dose at $5 \%$ of PTV

Using the calculated conformity and homogeneity indices according the RTOG protocol, we evaluated the TP that conforms more to PTV and is more homogeneous. The RTOG protocol defines the range of conformity and homogeneity as follows:

- If CI value is between 1 and 2; then, the treatment is in accordance with the protocol.

- If CI value is between 2 to 2.5 and 0.9 to 1 ; then, there is a minor deviation of the protocol.

- If the CI value is $>2.5$ and $<0.9$, it is considered as a severe deviation from the protocol.

For homogeneity, the ideal value for $\mathrm{HI}$ is 1 and it increases as the plan becomes less homogeneous. Values closer to 1 are more homogeneous than values away from 1 . The mean doses reaching the rectum, bladder, Right, and left femoral heads were also analyzed for each plan.

\section{Statistical Analysis}

A two-tailed pair $\mathrm{t}$-test was used to compare the mean of the different TPs at critical significant value of $5 \%$.

\section{Results}

In this study, the dose distribution for IMRT plan is more aligned to PTV than that of 3D CRT plan (as shown in Figure 1), which, in turn, reduces the dose to OAR. The dose coverage for both 3D-CRT and the IMRT TPs met the required criteria of at least $95 \%$ of the prescribed dose of PTV. The dose maximum was in the range of $105.5 \%-108 \%$ for $3 \mathrm{D}$-CRT plans, 
although it was one of the plans that had up to $108 \%$, which was due to the large size of the PTV. However, the dose maximum for IMRT was in the range of $104.5 \%-106.7 \%$.

Figure 2 shows the DVH of patients planned with 3D-CRT (left) and IMRT (right) treatment techniques, comparing their PTVs. The square box shows the PTV coverage of the TP done using IMRT technique, while the triangular shape is the PTV coverage of the TP done using $3 \mathrm{D}$-CRT TP technique.

\section{HI}

Results from the HI, H1, for the ten patients planned with 3D-CRT were in the range of 1.069-1.170, with an average of $1.088 \pm 0.03$. For IMRT, HI were in the range of 1.056-1.102, with an average of $1.072 \pm 0.002$. Also, $\mathrm{HI}\left(\mathrm{H}_{2}\right)$ for 3D-CRT were in the range of 1.029-1.128, with an average of $1.062 \pm 0.04$. However, for IMRT, HI

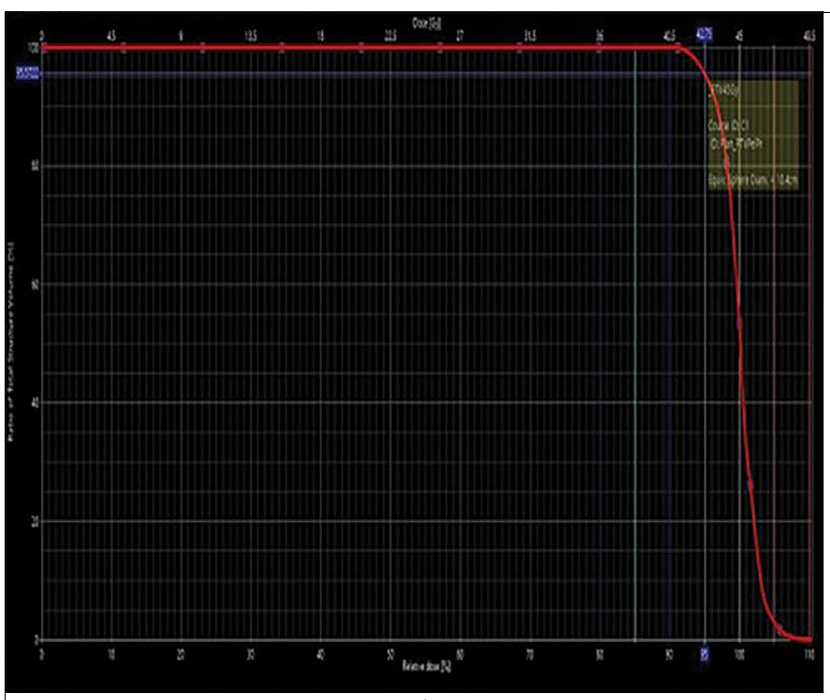

3D-CRT plan

$\left(\mathrm{H}_{2}\right)$ were in the range of $1.021-1.069$, with an average of $1.044 \pm 0.02$.

\section{CI}

The CI for each TP was calculated using equation 1. Figure 2 shows the dose coverage from the DVH. Table 2 shows the comparison between the CI of 3D-CRT and IMRT.

\section{OAR}

The dose to OAR of each patient planned using 3DCRT was compared to that of IMRT, as shown in the DVH in Figure 3. The DVH shows the dose to the rectum (brown), bladder (purple), left femoral head (blue), and right femoral head (sky-blue) for both TP. Tables 2 shows the mean results of dose to OAR for 3D-CRT and IMRT.

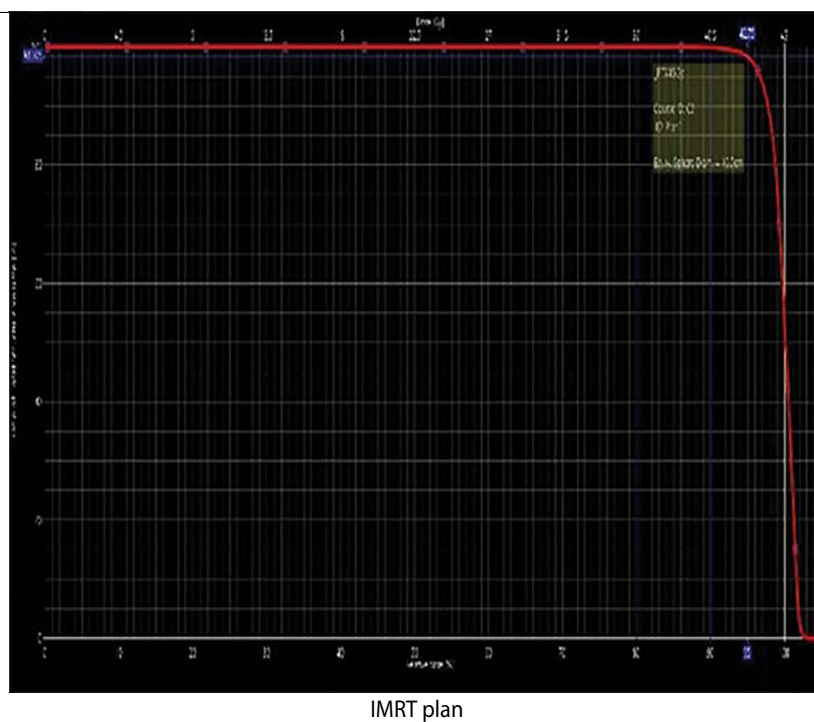

Fig. 2. Comprasion between the PTV coverage of the plan sum of 3D-CRT and IMRT plan.

Table 2 Comparison between the Organ at Risk for 3D-CRT and IMRT plans. V20 (Gy) and V50 (Gy) represents the dose to $20 \%$ volume and $50 \%$ volume of the OAR respectively. (QUANTEC)

\begin{tabular}{|c|c|c|c|c|}
\hline \multirow[t]{2}{*}{ Organs At Risk } & \multicolumn{2}{|c|}{ 3D-CRT } & \multicolumn{2}{|c|}{ IMRT } \\
\hline & V20 (Gy) & V50 (Gy) & V20 (Gy) & V50 (Gy) \\
\hline Rectum & $66.48 \pm 4.30 \mathrm{~Gy}$ & $53.76 \pm 4.00 \mathrm{~Gy}$ & $54.68 \pm 6.70 \mathrm{~Gy}$ & $42.25 \pm 4.90 \mathrm{~Gy}$ \\
\hline Bladder & $61.21 \pm 8.20 \mathrm{~Gy}$ & $52.39 \pm 5.10 \mathrm{~Gy}$ & $49.12 \pm 8.90 \mathrm{~Gy}$ & $31.44 \pm 9.40 \mathrm{~Gy}$ \\
\hline Femoral-Head L & $43.12 \pm 3.40 G y$ & $35.85 \pm 5.80 \mathrm{~Gy}$ & $33.53 \pm 4.50 \mathrm{~Gy}$ & $22.00 \pm 12.40 \mathrm{~Gy}$ \\
\hline Femoral-Head R & $36.02 \pm 8.50 \mathrm{~Gy}$ & $38.10 \pm 3.60 \mathrm{~Gy}$ & $33.42 \pm 4.10 \mathrm{~Gy}$ & $21.97 \pm 12.60 \mathrm{~Gy}$ \\
\hline
\end{tabular}

3D-CRT: Three-dimensional conformal radiotherapy ; IMRT: Intensity modulated radiotherapy; OAR: Organs at risk; QUANTEC: The Quantitative Analysis of Normal Tissue Effects in the Clinic. 


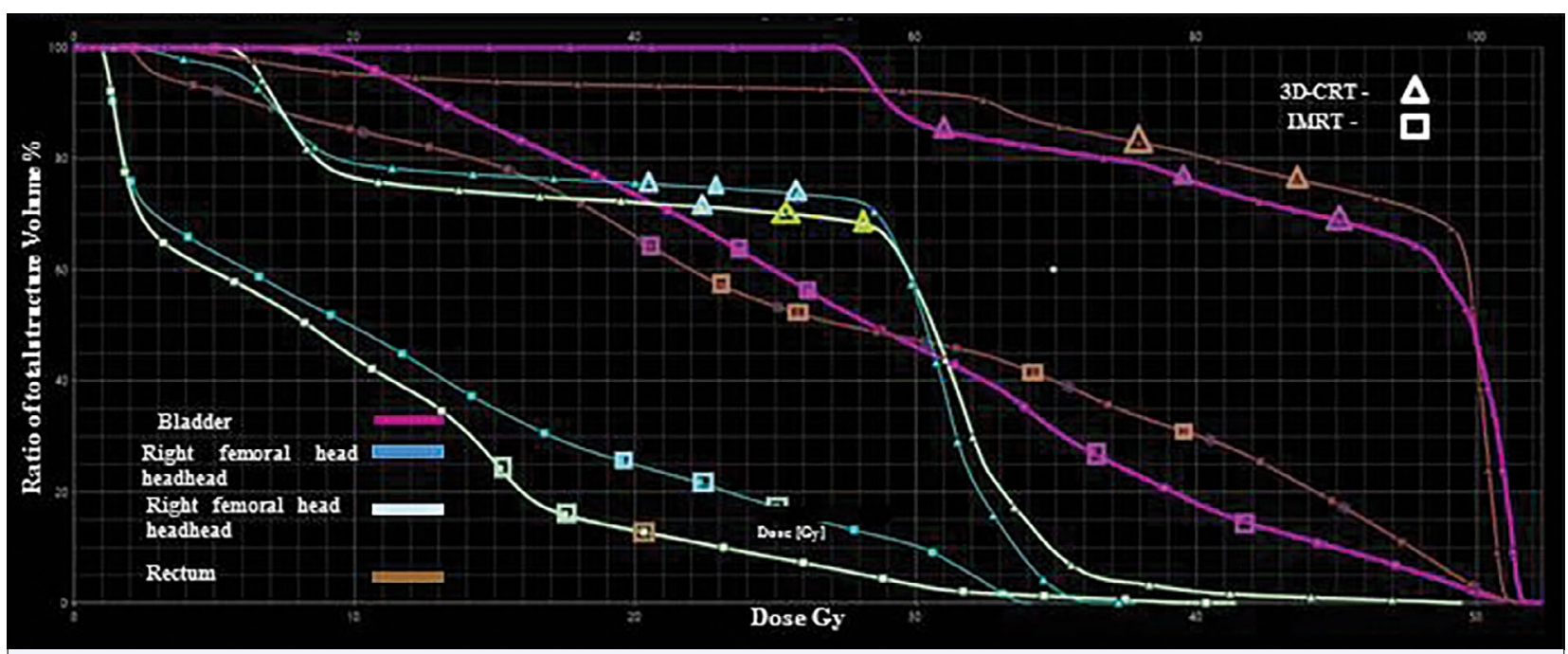

Fig. 3. DVH of OAR for 3D-CRT and IMRT treatment planning tecniques for one patient.

\section{Discussion}

In the treatment of cancer, sparing of OAR is one of the goals of radiotherapy. This was considered in this study. Both techniques were evaluated for sparing of OAR using the plan sum of the three phases. This study was aimed at comparing 3D-CRT and IMRT TPs in the treatment of neoplasm of prostate by comparing their HI, CI, and dose to OAR. The results from this study (Tables 2) show that IMRT is much better than 3D-CRT in terms of sparing of OAR. For 3D-CRT, it was observed that it was difficult to meet the RTOG dose constraint protocol for rectum, since the dose reaching $50 \%$ volume of the rectum was more than 50 Gy in most cases (Table 2); however, most of the plans met the QUANTEC protocol of $20 \%$ of the volume receiving 70 Gy (Table 2). For IMRT, the dose to OAR was within the tolerance set by RTOG and QUANTEC (Tables 2).

Table 2 shows the comparison between the OAR of 3D-CRT and that of IMRT. V20 (Gy) and V50 (Gy) represents the dose to $20 \%$ and $50 \%$ volume of OAR, respectively (QUANTEC). There was $21 \%$ reduction in dose to $20 \%$ volume of the rectum in IMRT and $27 \%$ reduction in dose to the $50 \%$ volume of the rectum in IMRT relative to the $3 \mathrm{D}$-CRT plans. $20 \%$ reduction in dose to $20 \%$ volume of the bladder and $40 \%$ reduction in $50 \%$ volume of the bladder in IMRT was also observed. More also, in the $20 \%$ volume of the right femoral head, there was $7.2 \%$ reduction and $42 \%$ reduction in the $50 \%$ volume of the right femoral head in the IMRT plans. The $20 \%$ of the left femoral head ex- perienced a $27 \%$ reduction in dose and $39 \%$ reduction in $50 \%$ volume of the dose received in the left femoral. These results were not comparable with other works because other studies evaluated different parameters.

Although several studies evaluated 3D-CRT and IMRT plans for single phase, this study paid more attention to plans of three phases and evaluation was done using their plan sum. More also, studies evaluating one and two phases were compared with our results. These studies adopted the HI defined by $\mathrm{Wu}$ et al.[12] In this study, the HI adopted was defined by RTOG protocol (defined as $\mathrm{H}_{1}$ ) and Yoon et al., (defined as $\mathrm{H}_{2}$ ), as stated in the materials and methods, and were compared using similar standard. From the result of this study (Table 3 ), HI $\left(\mathbf{H}_{1}\right)$ for IMRT showed a better homogeneity when compared to that of 3DCRT ( $p$-value $=0.03$ ). This result was close to that of $\mathbf{H}_{2}$ (Table 3); however, there was no statistically significant difference between the two techniques ( $p$-value $=0.16$ ). By relating the two results got from both protocols, it was discovered that the HI formula defined by Yoon et al., was closer to 1 than the RTOG protocol, since $\mathrm{HI}$ closer to 1 is the baseline for good homogeneity according to both protocols. Also, in this study, the result of CI (Table 3 ) shows that the conformity of IMRT (0.99) was better than that of 3D-CRT plans (0.91), such that it had a conformity closer to 1 than that of 3D-CRT. There was a statistically significant difference between the mean of both plans ( $p$-value $=0.23$ ). Compared to the study of Crowe et al.,[13] this CI of this study was closer to 1 when using the RTOG protocol. This was consistent with the study by Cristofaro et 
Table 3 Summary of results from this study

\begin{tabular}{|c|c|c|c|}
\hline TECHNIQUE & 3D-CRT & IMRT & $\mathbf{p}$ \\
\hline Number of patients & 10 & 10 & \\
\hline Max Total Volume of PTV & $1488 \mathrm{~cm}^{3}$ & $1488 \mathrm{~cm}^{3}$ & \\
\hline Mean Total Volume of PTV & $645.8 \mathrm{~cm}^{3}$ & $645.8 \mathrm{~cm}^{3}$ & \\
\hline Min Total volume of PTV & $96.2 \mathrm{~cm}^{3}$ & $96.2 \mathrm{~cm}^{3}$ & \\
\hline Mean Homogeneity index $\left(\mathrm{H}_{1}\right) \pm$ mean deviation & $1.088 \pm 0.03$ & $1.07 \pm 0.002$ & 0.03 \\
\hline Mean Homogeneity index $\left(\mathrm{H}_{2}\right) \pm$ mean deviation & $1.062 \pm 0.04$ & $1.044 \pm 0.02$ & 0.16 \\
\hline Conformity index $(\mathrm{Cl}) \pm$ mean deviation & $0.91 \pm 0.39$ & $0.99 \pm 0$ & 0.23 \\
\hline \multicolumn{4}{|l|}{ Rectum (mean values \pm mean deviation) } \\
\hline$D_{\max }(G y)$ & $77.64 \pm 29.09 \mathrm{~Gy}$ & $76.75 \pm 23.11 \mathrm{~Gy}$ & 0.60 \\
\hline$D_{\text {mean }}(G y)$ & $60.83 \pm 441.74 \mathrm{~Gy}$ & $44.22 \pm 180.50 \mathrm{~Gy}$ & 0.18 \\
\hline V35 (\%) & $92.47 \pm 434.03$ & $73.92 \pm 785.17$ & 0.45 \\
\hline V40 (\%) & $90.29 \pm 725.42$ & $60.77 \pm 757.50$ & 0.09 \\
\hline V50 (\%) & $57.48 \pm 3366.9$ & $29.97 \pm 646.8$ & 0.09 \\
\hline \multicolumn{4}{|l|}{ Bladder (mean values \pm mean deviation) } \\
\hline $\mathrm{D}_{\max }(\mathrm{Gy})$ & $78.02 \pm 22.44$ & $78.28 \pm 22.54$ & 0.86 \\
\hline$D_{\text {mean }}(G y)$ & $56.79 \pm 281.54$ & $42.14 \pm 88.73$ & 0.009 \\
\hline V35 (\%) & $91.94 \pm 267.23$ & $62.31 \pm 337.60$ & 0.00065 \\
\hline V40 (\%) & $93.56 \pm 379.11$ & $46.82 \pm 495.98$ & 0.0001 \\
\hline V50 (\%) & $59.17 \pm 3469.7$ & $24.86 \pm 626.22$ & 0.043 \\
\hline \multicolumn{4}{|l|}{ Right Femoral head (mean values \pm mean deviation) } \\
\hline$D_{\max }(G y)$ & $51.24 \pm 250.6 \mathrm{~Gy}$ & $47.85 \pm 250.56 \mathrm{~Gy}$ & 0.52 \\
\hline$D_{\text {mean }}(G y)$ & $33.18 \pm 330.4 \mathrm{~Gy}$ & $20.76 \pm 497.34 \mathrm{~Gy}$ & 0.89 \\
\hline V35 (\%) & $31.16 \pm 2857.7$ & $13.36 \pm 1390.0$ & 0.157 \\
\hline V40 (\%) & $34.40 \pm 6104.6$ & $10.28 \pm 730.92$ & 0.308 \\
\hline V50 (\%) & $5.39 \pm 251.40$ & $0.35 \pm 2.45$ & 0.25 \\
\hline \multicolumn{4}{|l|}{ Left Femoral head (mean values \pm mean deviation) } \\
\hline $\mathrm{D}_{\max }(\mathrm{Gy})$ & $52.65 \pm 204.89 \mathrm{~Gy}$ & $37.63 \pm 1262.5 \mathrm{~Gy}$ & 0.117 \\
\hline$D_{\text {mean }}(G y)$ & $35.23 \pm 304.04 \mathrm{~Gy}$ & $21.08 \pm 422.4 \mathrm{~Gy}$ & 0.468 \\
\hline V35 (\%) & $69.63 \pm 1627.5$ & $30.52 \pm 2196.7$ & 0.022 \\
\hline V40 (\%) & $36.86 \pm 5510.2$ & $13.91 \pm 1363.0$ & 0.25 \\
\hline V50 (\%) & $6.61 \pm 24.82$ & $1.11 \pm 24.82$ & 0.39 \\
\hline
\end{tabular}

3D-CRT: Three-dimensional conformal radiotherapy; IMRT: Intensity modulated radiotherapy PTV: Planning target volume.

al.[14] and Jamal, et al.[15] The result from this study contradicts that of Kinhkikar, et al.,[16] since their CIs were $0.97 \pm 0.02$ and $0.98 \pm 0.02$ for IMRT and 3D-CRT, respectively, thus resulting in a better conformity in 3D-CRT than in IMRT. This may be due to the level of experience of the IMRT planner.

In this study, the mean dose to the left femoral head was reduced by $40.2 \%$ in IMRT. This was consistent with the study by Uysal et al.,[17] who reported a mean dose of $18.79 \pm 18.79$ and $31.5 \pm 4.11$ Gy for IMRT and 3D-CRT, respectively, thus resulting in $40.3 \%$ reduction. This was also consistent with the study by Cristofaro, et al., and Crowe et al. In Table 3, the volume of the bladder receiving 35 Gy (V35) had 20.1\% reduction in IMRT and this result was close to the result of Kinhikar et al., with 23.7\% reduction in IMRT for V35. The volume of the bladder receiving $40 \mathrm{~Gy}$ had a reduction of $49.9 \%$ reduction in IMRT relative to 3D-CRT. This was higher than the $41 \%, 37.61 \%, 24.7 \%$, and $26.8 \%$ reported by Cristofaro, et al., Ashman et al.,[18] Uysal et al., and Kinhikar et al., respectively. For the rectum, the volume receiving 40 Gy had a $32.7 \%$ reduction in IMRT relative to 3DCRT. Crowe et al., had a reduction of $49 \%$ in the volume receiving $40 \mathrm{~Gy}$ in IMRT, while $50 \%$ reduction was reported by Kinhikar et al. However, Cristofaro, et al., had 34\% reduction, which is closer to our result. Other studies by Wortel et al.[19] and Panayiotis et al.[20] also had reduction in IMRT.

Generally, the results from this study were comparable to that of other studies; however, homogeneity and conformity indices were better and had lesser dose to OAR. 


\section{Conclusion}

Twenty TPs of 3D-CRT and IMRT were created and their $\mathrm{CI}$ and $\mathrm{HI}$ were evaluated for ten prostate patients. Also, the dose to OAR was evaluated. The use of IMRT TP technique for prostate cancer proved to be superior over 3D-CRT and in sparring dose to OAR. More also, the control of normal tissue complication probability is better with plans done in more than one phases compared to those done in a single phase.

Acknowledgement: The authors would like to appreciate the management of NSIA-LUTH Cancer Care (NLCC) Lagos, Nigeria for granting us the permission to use the center for this study.

Peer-review: Externally peer-reviewed.

Conflict of Interest: All authors declared no conflict of interest.

Ethics Committee Approval: This study was approved by the College of Medicine University of Lagos Ethics Committee (no. 17758, date: 06/08/2018).

Financial Support: None declared.

Authorship contributions: Concept - S.A., M.A., M.H., A.O., A.J.; Design - M.A., A.D., A.O., S.A., M.A.; Supervision - S.A., M.A., M.A., M.H., J.A.; Funding - None; Materials - A.D., M.A.; Data collection and/or processing - J.A., A.J., A.O., M.A.; Data analysis and/or interpretation - A.J., A.O., S.A., M.A.; Literature search - M.A., A.D.; Writing S.A., M.A., M.H., A.O., A.J., J.A., A.D., M.A.; Critical review - S.A., M.A., M.H., A.O., A.J., J.A., A.D., M.A.

\section{References}

1. American Cancer Society: available at: https://www. cancer.org/research/cancer-facts-statistics/all-cancerfacts-figures/cancer-facts-figures-2019.html. Accessed Jun 8, 2020.

2. The International Agency for Research on Cancer: The Global Cancer Observatory. 2019. Available at: https:// gco.iarc.fr/. Accessed Jun 8, 2020.

3. Nigeria Health Blog. Available at: https://nimedhealth. com.ng/2019/02/05/prostate-cancer-in-nigeria-epidemiology-awareness-screening-symptoms-treatment. Accessed Jun 8, 2020.

4. Hou H, Swanson D, Barqawi AB. Modalities for Imaging Prostate Cancer. Hindawi Publishing Corporation Advances in Urology 2009;65:12.

5. Quantitative Analysis of Normal Tissue Effects in Clinic (QUANTEC). International Journal of Radiation Oncology, Biology and Physics 2010;76(Sup$\mathrm{pl}): S 3-S 9$.
6. ICRU. Prescribing, Recording, and Reporting Intensity-Modulated Photon-Beam Therapy (IMRT)(ICRU Report 83). Journal of International Commission on Radiation Units and Measurements 2010;10:1-106.

7. Viswanathan AN, Yorke ED, Marks LB. Radiation dose-volume effects of the urinary bladder. International Journal of Radiation Oncology, Biology and Physics 2010;76(Suppl. 3):S116-22

8. Michalski JM, Gay H, Jackson A, Tucker SL, Deasy JO. Radiation dose-volume effects in radiation-induced rectal injury. International Journal of Radiation Oncology, Biology and Physics. 2010;76(3 Suppl):S123-9.

9. Eifel PJ, Winter K, Morris M, Levenback C, Grigsby $\mathrm{PW}$, Cooper J, et al. Pelvic irradiation with concurrent chemotherapy versus pelvic and para-aortic irradiation for high-risk cervical cancer: an update of radiation therapy oncology group trial (RTOG) 90-01. J Clin Oncol 2004;22(5):872-80.

10. Knoos T, Kristensen I, Nilsson P. Volumetric and dosimetric evaluation of radiation treatment plans: radiation conformity index. Journal of Radiation Oncology, Biology, Physics 1998;42(5):1169-76.

11. Yoon M, Park SY, Shin D, Lee SB, Pyo HR, Kim DY. A new homogeneity index based on statistical analysis of the dose-volume histogram; Journal of Applied Clinical Medical Physics 2007;8(2):9-17.

12. Wu VW, Kwong DL, Sham JS. Target dose conformity in 3-dimensional conformal radiotherapy and intensity modulated radiotherapy. Radiotherapy Oncology 2004:71(2):201-6.

13. Crowe SB, Kairn T, Middlebrook N, Hill B, Christie DR, Knight RT, et al. Retrospective evaluation of dosimetric quality for prostate carcinomas treated with 3D conformal, intensity modulated and volumetric modulated arc radiotherapy. J Med Radiat Sci 2013;60(4):131-8.

14. Cristofaro N, Hindson B, Sanderson C. Retropective dosimetric comparison of three-dimensional conformal radiotherapy (3D-CRT), sliding window intensity modulated radiotherapy (IMRT) and volumetric modulated arc therapy (VMAT) for protate cancer. 2014, R-0144. Available at: https://epos.myesr.org/poster/ ranzcr/ranzcr2014/R-0144. Accessed Jun 8, 2020.

15. AL-Shareef JM, Attalla EM, Khalil MM, Abdelaal AM, El-Nagdy MS. A comparison of intensity modulated and 3-Dimensional conformal radiotherapy for prostate cancer using 6-MV and 15-MV photon energies. Arab Journal of Nuclear Science and Applications. 2020;53(2):189-200.

16. Kinhikar RA, Pawar AB, Mahantshetty U, Murthy V, Dhehpande DD, Shrivastava SK. Rapid Arc, helical tomotherapy, sliding window intensity modulated radiotherapy and three dimensional conformal radiation for localized prostate cancer: A dosimetric com- 
parison. Journal of Cancer Resaerch and Therapeutics 2014;10(3):575-82.

17. Uysal B, Beyzadeoglu M, Sager O, Dincoglan F, Demiral S, Gamsiz H, et al. Dosimetric evaluation of intensity modulated radiotherapy and 4 -Field 3D-confromal radiotherapy in prostate cancer. Balkan Medical Journal 2013;30:54-7.

18. Ashman JB, Zelefsky MJ, Hunt MS, Leibel SA, Fuks $\mathrm{Z}$. Whole pelvic radiotherapy for prostate cancer using 3D conformal and intensity-modulated radiotherapy; International Journal of Radiation Oncology Biology
Physics 2005;63(3):765-71.

19. Wortel R, Incrocci L, Pos F, Van Der Heide U, Lebesque J, Aluwini S, et al. PO-0742: Image-guided IMRT reduces late toxicity compared to 3D-CRT for prostate cancer. Radiotherapy and Oncology 2016;119(Supplement 1):S346-S7.

20. Mavroidis P, Komisopoulos G, Buckey C, Mavroeidi M, Swanson GP, Baltas D, et al. Radiobiological evaluation of prostate cancer IMRT and conformal-RT plans using different treatment protocols. Phys Med 2017;40:33-41. 